\title{
Existence and Fairness of Value Allocation without Convex Preferences
}

\author{
Nicholas C. YanNelis* \\ Department of Economics, Wayne State University, \\ Detroit, Michigan 48202
}

Received March 31, 1982; revised July 26, 1982

\begin{abstract}
Shafer (Econometrica 48 (1980), 467-476) proved that in a finite exchange economy value allocations exist, provided that each agent has convex, complete, transitive, compact and monotone preferences. However, if preferences are not convex, then value allocations may not exist. To remedy this difficulty we enlarge the set of value allocations by introducing the concept of approximate value allocations, and show that in a finite exchange cconomy approximatc valuc allocations exist, even if preferences are not convex, or compact, or monotone. This value existence result can be used to provide a very general value existence theorem for a sequence of finite economies. Further, we show that value allocations may discriminate in favor of or against a coalition of agents. Journal of Economic Literature Classification Numbers: 020, 021, 022.
\end{abstract}

\section{INTRODUCTION}

Shapley $[6]$ showed that in a finite exchange economy if the set of all attainable utility vectors (i.e., the Pareto set) is convex and compact, then cardinal ${ }^{1}$ value allocations exist. It can easily be shown that the above result guarantees the existence of ordinal value allocations as well. However, Kannai and Mantel $[4]$ showed that in a finite exchange economy with convex and monotone preferences for each agent, the Pareto set will not be convex for any utility function choice. Thus, as a consequence, value allocations may not exist. Shafer [8], however, assuming convex and monotone preferences for each agent and using a class of utility functions known as "minimum income functions," 2 was able to prove the existence of ordinal value allocations even if the Pareto set was not convex.

* This paper is based on a chapter of my Ph.D thesis a the University of Rochester under the supervision of Professors M. Ali Khan and Lionel W. McKenzie, to whom I am heavily indebted. Thanks are also due to Allen Scafuri and to a careful referee for some useful remarks.

${ }^{1}$ See $|8|$ and its reference for a distinction between ordinal and cardinal value allocations.

${ }^{2}$ This will be defined in Section 2.3. 
It is the purpose of the present paper first to improve further the ordinal value allocations existence theorem by allowing not only for a nonconvex Pareto set but also for nonconvex and nonmonotone preferences for each agent. To this end we enlarge the set of value allocations by introducing the concept of approximate value allocations and show that in a finite exchange economy approximate value allocations exist. Second, we show that value allocations do not satisfy an equity criterion named coalitional fairness of allocations. Although our existence theorem was inspired from the elegant proof given in Shafer [8], we employ much weaker assumptions. In particular, we need not have either convex, or compact, or monotone preferences. The relaxation of these assumptions requires a proof of a novel type. However, we note that the recent nonstandard existence proof for a competitive equilibrium by Khan and Rashid $[3 \mid$ and its translation by Anderson et al. [1] proved very useful in carrying out the arguments.

The cost that we pay for adopting very weak assumptions is that value allocations do not satisfy the requirement that the aggregate exccss demand is zero. However, aggregate excess demand does have an upper bound. We should emphasize that it turns out that the bound depends on the norm of endowments, the square root of the number of agents in the economy, and a constant number of commodities. In fact, since preferences are not compact the bound we derive is independent of preferences and can be easily computed.

Finally, it should be emphasized that the advantage of considering a finite exchange economy is that it provides a computable bound or error on aggregate excess demand. Consequently, we always know how far approximate value allocations are from value allocations. In contrast, if one considers "large" economies ${ }^{3}$ as in Shapley and Shubik |7|, then the approximate notions are defined with respect to an arbitrary small positive number, i.e., $\varepsilon$. Thus, all one could say in the context of a large but finite economy is that $\varepsilon$ is a small positive number which tends to zero as the number of agents in the economy goes to infinity. Consequently, in a large but finite economy, approximate or $\varepsilon$-value allocations will tend to value allocations as the economy becomes infinite. ${ }^{4}$ In order to indicate the relationship between the fixed economy formulation and the sequential formulation, we will show how our value existence theorem can be used to provide a very general value existence result for a sequence of finite economies.

Section 2 of the paper presents the model and results; their proofs are concentrated in Section 3.

\footnotetext{
${ }^{3}$ That is, sequences of finite economies.

${ }^{4}$ Alternatively we could say that as the number of agents in the economy goes to infinity, the aggregate excess demand tends to zero.
} 


\section{The Model AND Results}

\subsection{Notation}

$\mathbb{R}$ denotes the set of real numbers.

$\mathbb{R}^{l}$ denotes the $l$-fold Cartesian product of $\mathbb{R}$.

$\mathbb{R}_{+}^{l}$ denotes the positive orthant of $\mathbb{R}^{\prime}$, i.e.,

$$
\mathbb{R}_{+}^{l}=\left\{x \in \mathbb{R}^{l}: x \geqslant 0\right\} .
$$

For any $x, y$ in $\mathbb{R}^{l}, x \geqslant y$ means $x_{i} \geqslant y_{i}$ for all $i=1,2, \ldots, l ; x>y$ means $x \geqslant y$ and $x \neq y ; x \gg y$ means $x_{i}>y_{i}$ for all $i$.

$$
\begin{aligned}
& e \in \mathbb{P}^{\prime} \text { denotes } e=(1,1, \ldots, 1) . \\
& |S| \text { denotes the number of elements in the set } S . \\
& \mathbf{P}(A) \text { denotes the set of subsets of } A .
\end{aligned}
$$

For any $A \subset \mathbb{R}^{l}$, con $A$ denotes the convex hull of $A$.

$\backslash$ denotes the set theoretic subtraction.

$$
\|x\|=\sum_{i=1}^{l}\left|x_{i}\right|
$$

$S(x, r)$ denotes the open ball in $\mathrm{R}_{+}^{l}$ of radius $r$ centered at $x$. For any $x, y$ in $\mathbb{R}^{l}, x \cdot y$ denotes the inner product, i.e.,

$$
x \cdot y=\sum_{i=1}^{l} x_{i} y_{i} \text {. }
$$

\subsection{Definitions}

Let $\mathscr{P}$ denote the set of completc, transitive, continuous and locally nonsatiated binary relations $\gtrsim$ on $\mathrm{R}_{+}^{l}$ (the commodity space).

A utility function $u$ for a binary relation $\gtrsim$ on $\mathbb{R}_{+}^{l}$ is a real valued function on $\mathbb{R}_{+}^{l}$ such that $u(x) \geqslant u(y) \Leftrightarrow x \gtrsim y$.

A finite exchange economy $\mathscr{E}$ is a mapping of $T$ into $\mathscr{P} \times \mathbb{R}_{+}^{l}$, where $T$ is a finite set of agents whose cardinality is $n$, i.e., $|T|=n$. Let $\gtrsim_{t}$ be the projection of $\mathscr{E}(t)$ onto $\mathscr{P}$ and $e(t)$ the projection of $\mathscr{E}(t)$ onto $\mathbb{R}_{+}^{l}$. We interpret $\gtrsim_{t}$ as the preference of agent $t$ and $e(t)$ as his initial endowment.

A coalition is a subset of the set of agents.

A game with side payments $G=(T, v)$ consists of a finite set of agents $T$ and a superadditive function $v$ of $\mathbf{P}(T)$ into $\mathbb{R}$ such that $v(\varnothing)=0$.

The Shapley value of the game $G$ assigns to each agent $t$ a value which 
shows the marginal contribution made by that agent to all the coalitions of which he is a member, i.e., $s_{t}=\sum_{s \subset r}((|S|-1) !(|T|-|S|) ! / T \mid !)$ $[v(S)-v(S \backslash\{t\})]$. In other words the Shapley value of the same $G$ assigned to an agent is given by summing up his dividends from the marginal value of each coalition in which he is a member.

Given a finite exchange economy $\mathscr{E}$ and a family $u=\left\{u_{t}\right\}_{t \in T}$ of utility functions representing the preferences $\left\{\gtrsim_{t}\right\}_{t \in T}$, define a game $\left(T, v_{u}\right)$ by

$$
\begin{aligned}
v_{u}(S)=\max _{x(t)}\left\{\sum_{t \in S} u(x(t), t): \sum_{t \in S} x(t)=\sum_{t \in S} e(t)\right. \\
\text { and } \left.x(t) \in \mathbb{R}_{+}^{l} \text { for all } t \in S\right\} .
\end{aligned}
$$

A function $x(t)$ of $T$ into $\mathbb{R}_{+}^{l}$ is said to be a value allocation for $\mathscr{E}$ if and only if :

(i) $\sum_{t \in T} x(t)=\sum_{t \in T} e(t)$

(ii) there exists a family $u=\left\{u_{t}\right\}_{t \in T}$ of utility functions representing the preferences $\left\{\gtrsim_{t}\right\}_{t \in T}$ such that for all $t \in T, u(x(t), t)$ is the Shapley value of the game $\left(T, v_{u}\right)$.

Hence, $x(t)$ is a value allocation for the finite exchange economy $\mathscr{E}$ if first, the aggregate excess demand is zero, and second, it yields to each agent a utility which is equal to his shares of the marginal value of each coalition in which he is a member.

\subsection{Approximate Value Allocations}

A price system $p$ is a vector in $\mathbb{R}^{l}$ such that $p \in S=\left\{x \in \mathbb{R}_{+}^{l}: \max x_{i} \leqslant 1\right.$, $\left.x_{i}>0, i=1,2, \ldots, l\right\}$. Let $M(x, p, t)$ be the minimum income function of agent $t$ (see McKenzie [5]), which allows him to achieve at the price vector $p$, a commodity combination at least as good as $x$, i.e., $M(x, p, t)=\min _{x^{\prime} \in C_{x}}$ $p \cdot x^{\prime}$, where $C_{x}=\left\{x^{\prime} \in \mathbb{R}_{+}^{l}: x^{\prime} \gtrsim_{t} x\right\}$. It is known (see, for instance, McKenzie [5], ${ }^{5}$ or Shafer $[8$, p. 469$\left.]\right)$ that $M(x, p, t)$ is continuous and represents a utility function for the preference $\gtrsim_{t}$.

Let $p$ be a price system, and consider the game $(T, v(p, \cdot))$ defined by $v(p, B)=\max _{x(t)}\left\{\sum_{t \in B} M(x, p, t): \sum_{t \in B} x(t)=\sum_{t \in B} e(t)\right.$ and $x(t) \in \mathbb{R}_{+}^{l}$ for all $t \in B\}$. Denote by $\operatorname{Sh}(p, t)$ the Shapley value of agent $t$ of the above game.

A function $x(t)$ of $T$ into $\mathbb{R}_{+}^{l}$ is said to be an approximate value allocation for $\mathscr{E}$ if there exists price system $p$ such that

${ }^{5}$ McKenzie [5, footnote 2] shows that $M(x, p, t)$ is concave in $p$ and consequently, continuous in $p$. 
(i) $M(x, p, t)=\operatorname{Sh}(p, t)$ for all $t \in T$;

(ii) $\sum_{i=1}^{l} \max \left\{\sum_{i \in T} x_{i}(t)-\frac{\Gamma}{i \in T} e_{i}(t), 0\right\} \leqslant(l+1) \sqrt{n} \max _{t \in T}\|e(t)\|$.

Condition (i) shows that there is a price system $p$ at which if each trader will minimize his expenditure over his preferred set then his utility is equal to his Shapley value. Condition (ii) says that the aggregate excess demand is bounded above by a bound which depends on the norm of the initial endowments, the square root of the number of agents and a constant number of commodities. Note that the above concept of value allocation is different from the one used in Shafer $[8$, p. 429]. In particular, due to nonconvex preferences for all agents the aggregate excess demand is not zero as in Shafer [8], but does have an upper bound. However, if the bound or error $(l+1) \sqrt{n} \max _{t \in T}\|e(t)\|$ is zero then both notions coincide.

\subsection{Coalitionally Fair Allocations}

A function $x(t)$ of $T$ into $\mathbb{R}_{+}^{l}$ is said to be an allocation if $\sum_{t \in T} x(t)=$ $\sum_{t \in T} e(t)$. Let $S_{1}, S_{2}$ be two disjoint coalitions. An allocation $x(t)$ is coalitionally fair ${ }^{6}$ (c-fair) if there exist no $y(t)$ and $i=1,2$ such that for all $t \in S_{i}, y(t)>_{t} x(t)$ and $\sum_{t \in S_{i}}(y(t)-e(t))=\sum_{t \in S_{j}}(x(t)-e(t)), j \neq i$. In other words, an allocation is said to be $c$-fair if no group of agents can redistribute among its members the net trade of any other group of agents and become better off. More simply, an allocation is $c$-fair if no coalition of agents envies the net trade of any other coalition of agents.

\subsection{Results}

THEOREM 1. An approximate value allocation in $\mathscr{E}$ exists.

THEOREM 2. A value allocation in $\mathscr{E}$ may not be $c$-fair.

THEOREM 3. Let $\mathscr{E}_{n}: T_{n} \rightarrow \mathscr{P} \times \mathbb{R}_{+}^{l}$ be a sequence of finite economies. If $\left|T_{n}\right| \rightarrow \infty$ and for any $S_{n} \subset T_{n}\left(\left|S_{n}\right| / \sqrt{\left|T_{n}\right|}\right) \rightarrow 0 \Rightarrow \| \sum_{s_{n}} e_{n}(t)|| / \sqrt{\left|T_{n}\right|} \rightarrow 0$, then there exist $x_{n}: T_{n} \rightarrow \mathbb{R}_{+}^{l}$ and $p_{n} \in S$ such that:

(i) $M\left(x_{n}, p_{n}, t\right)=\operatorname{Sh}\left(p_{n}, t\right) \quad$ for all $t \in T_{n}$.

(ii) $\frac{1}{\left|T_{n}\right|} \sum_{i=1}^{l} \max \left\{\sum_{t \in T_{n}}^{\searrow} x_{n}^{i}(t)-\sum_{t \in T_{n}}^{\searrow} e_{n}^{i}(t), 0\right\}$

$$
\leqslant \frac{1}{\sqrt{\left|T_{n}\right|}}(l+1) \max _{t \in T_{n}}\left\|e_{n}(t)\right\| \rightarrow 0
$$

\footnotetext{
${ }^{6}$ This concept of $c$-fair allocations was explored in $[2,10]$, where we refer the reader to it's relationship to the literature on fairness.
} 
Theorem 2 shows that a value allocation may discriminate in favor of or against some coalitions of agents. In other words, at a value allocation a group of agents can redistribute among its members the net trade of another group of agents and become better off.?

Theorem 3 shows the existence of an approximate value allocation for a sequence of finite economies provided that initial endowments are integrable, i.c., no "small" group of agents can hold sufficiently large endowments. ${ }^{8}$ Conclusion (i) is the same as that in Theorem 1. Conclusion (ii) shows that as the number of agents goes to infinity, the average excess demand goes to zero. Consequently, the average demand approximately balances the average supply with the approximation getting better the larger the finite economy.

\section{Proofs}

Proof of Theorem 1. In order to prove Theorem 1 we compactify the excess demand space by a bound which depends on the number of agents in the economy and the initial endowments; and we let the price space vary with the number of agents in the economy. It should be noted that since our bounds are chosen independently of the degree of nonconvexity of preferences, the approximating procedure will give rise to a computable error on aggregate excess demand.

Note first that, $M(x, p, t)=p \cdot x$ whenever $p$ is normal to a plane of support of $C_{x}$. Moreover, by individual rationality, ${ }^{9} \operatorname{Sh}(p, t) \geqslant$ $M(e(t), p, t) \geqslant 0$ for all $t \in T$.

Consider the set valued functions:

$$
\begin{aligned}
& H(p, t)=\left\{x \in \mathbb{R}_{+}^{\prime}: p \cdot x \leqslant \operatorname{Sh}(p, t)\right\}, \\
& D(p, t)=\left\{x \in \mathbb{R}_{+}^{\prime}: x \in H(p, t) \text { and } x \text { is maximal for } z_{t} \text { in } H(p, t)\right\} .
\end{aligned}
$$

Let

$$
\begin{aligned}
& e=\sum_{i \in T} e(t), \\
& x=\sum_{t \in T} x(t),
\end{aligned}
$$

\footnotetext{
${ }^{7}$ It should be remarked that the competitive equilibrium is $c$-fair (see $\left.|2|\right)$.

${ }^{8}$ The integrability assumption can be weakened to $\max _{t \in T_{n}} \| e_{n}(t) \mid / \sqrt{\left|T_{n}\right|} \rightarrow 0$. The latter assumption rules out dominant agents, i.e., monopolists or oligopolists, however, it allows for dominant classes of individuals.

${ }^{9}$ Individual rationality and group rationality are two of the properties of the Shapley Value, i.e., $\operatorname{Sh}(p, t) \geqslant M(e(t), p, t)$ and $\sum_{t \in T} \operatorname{Sh}(p, t)=v(p, T) \leqslant p \cdot \sum_{T} e(t)$; see also Shafer 18 . p. 474 |.
} 


$$
\begin{aligned}
D(p) & =\sum_{t \in T}^{\urcorner} D(p, t), \\
\zeta(p) & =D(p)-e, \\
Z & =\mathbb{R}_{+}^{\prime}-\{e\}, \\
z & =x-e .
\end{aligned}
$$

Obviously, $z$ is a point in $\zeta(p) \subset Z$.

Let

$$
Z^{\prime}=\left\{z \in Z:\|z\| \leqslant n \max _{t \in \Gamma}\|e(t)\|\right\}
$$

Define

$$
\begin{aligned}
\Delta & =\left\{p \in S: p_{i} \geqslant \frac{1}{\sqrt{n}}, i=1,2, \ldots, l\right\}, \\
\varphi(z) & =\{p \in \Delta: p \cdot z=\max q \cdot z \text { for all } q \in \Delta\}, \\
\Theta(p, z) & =\varphi(z) \times \operatorname{con} \zeta(p) .
\end{aligned}
$$

Thus, $\Theta$ is a set valued function from the set $\Delta \times Z^{\prime}$ into itself. Standard arguments can be applied to show that the mapping $\Theta$ satisfies all the properties of the Kakutani fixed point theorem. Consequently, there exist $(p, z) \in \Theta(p, z)$, i.e., $z \in \operatorname{con} \zeta(p)$ and for all $q \in A, q \cdot z \leqslant p \cdot z \leqslant 0$. By the Corollary of the Shapley-Folkman theorem (Starr $[9$, p. 35]) we can write: $z=\sum_{t \in T^{\prime}} x(t)+\sum_{i=1}^{l} x^{\prime}\left(t_{i}\right)-\sum_{t \in T} e(t)$, where $T^{\prime}=T \backslash\{1,2, \ldots, l\}$ and $x(t) \in$ $D(p, t)$ for all $t \in T^{\prime}$ and $x^{\prime}\left(t_{i}\right) \notin D\left(p, t_{i}\right)$ for all $i=1, \ldots, l$. Pick $x\left(t_{i}\right)$ so that $x\left(t_{i}\right) \in D\left(p, t_{i}\right)$ for all $i=1,2, \ldots, l$. Then

$$
\begin{aligned}
z\left(t_{i}\right)-z^{\prime}\left(t_{i}\right) & =\left(x\left(t_{i}\right)-x^{\prime}\left(t_{i}\right)\right)-\left(e\left(t_{i}\right)-e^{\prime}\left(t_{i}\right)\right) \\
& =z\left(t_{i}\right)+e\left(t_{i}\right)-\left(z^{\prime}\left(t_{i}\right)+e\left(t_{i}\right)\right) \\
& \leqslant z\left(t_{i}\right)+e\left(t_{i}\right) .
\end{aligned}
$$

Hence,

$$
\begin{aligned}
\sum_{j=1}^{\ell} \max \left\{z_{j}\left(t_{i}\right)-z_{j}^{\prime}\left(t_{i}\right), 0\right\} & \leqslant \sum_{j=1}^{l}\left(z_{j}\left(t_{i}\right)+e_{j}\left(t_{i}\right)\right) \\
& \leqslant \frac{p \cdot e\left(t_{i}\right)}{\min \left(p_{1}, \ldots, p_{l}\right)} \\
& \leqslant \sqrt{n}\left\|e\left(t_{i}\right)\right\| .
\end{aligned}
$$


Moreover,

$$
\begin{aligned}
q \cdot\left[\sum_{i \in T} x(t)-\sum_{t \in T} e(t)\right]= & q \cdot\left[\sum_{i=1}^{l} x\left(t_{i}\right)-\sum_{i=1}^{l} e\left(t_{i}\right)\right] \\
& -q \cdot\left[\sum_{i=1}^{l} x^{\prime}\left(t_{i}\right)-\sum_{i=1}^{l} e^{\prime}\left(t_{i}\right)\right]+q \cdot z .
\end{aligned}
$$

From (2) and (3) it follows that for all $q \in \Delta$

$$
q \cdot\left[\sum_{t \in T} x(t)-\sum_{t \in T} e(t)\right] \leqslant l \sqrt{n} \max _{t \in T}\|e(t)\| .
$$

Let

$$
\begin{aligned}
q^{i}= & 1 \text { if }\left(\sum_{i \in T} x(t)-\sum_{t \in T} e(t)\right)^{i}>0, \quad i=1, \ldots ., \\
& \frac{1}{\sqrt{n}} \text { if }\left(\sum_{t \in T} x(t)-\sum_{t \in T} e(t)\right)^{i} \leqslant 0, \quad i=1, \ldots, l
\end{aligned}
$$

Substituting (5) into (4), we have

$$
\begin{aligned}
& q \cdot\left[\sum_{t \in T} x(t)-\sum_{t \in T} e(t)\right]=\sum_{\text {some } i}\left[\frac{\bigvee}{i \in T} x_{i}(t)-\sum_{t \in T} e_{i}(t)\right] \\
& +\frac{1}{\sqrt{n}} \underset{\text { some } i}{\searrow}\left[\frac{V}{t \in T} x_{i}(t)-\sum_{t \in T} e_{i}(t)\right] \\
& \leqslant l \sqrt{n} \max _{t \in T}\|e(t)\| \text {. }
\end{aligned}
$$

Hence,

$$
\begin{aligned}
& \sum_{i=1}^{\prime} \max \left\{\sum_{t \in T} x_{i}(t)-\bigvee_{t \in T} e_{i}(t), 0\right\} \\
& \leqslant l \sqrt{n} \max _{t \in T}\|e(t)\|-\frac{1}{\sqrt{n}} \underset{\operatorname{somc} i}{\searrow}\left[\frac{\searrow}{i \in T} x_{i}(t)-\frac{\bigvee}{\epsilon \in T} e_{i}(t)\right] \\
& =l \sqrt{n} \max _{t \in T}\|e(t)\|+\frac{1}{\sqrt{n}} \underset{\text { some } i}{\longleftarrow}\left[\left|\sum_{t \in T} x_{i}(t)-\underset{t \in T}{\bigvee} e_{i}(t)\right|\right] \\
& \leqslant l \sqrt{n} \max _{t \in T}\|e(t)\|+\frac{1}{\sqrt{n}} \sum_{i=1}^{l}\left[\left|\frac{\sum_{t \in T}}{i} x_{i}(t)-\sum_{t \in T} e_{i}(t)\right|\right]
\end{aligned}
$$




$$
\begin{aligned}
& =l \sqrt{n} \max _{t \in T}\|e(t)\|+\frac{1}{\sqrt{n}}\left\|\frac{\sum_{t \in T}}{} x(t)-\frac{\Gamma}{i \in T} e(t)\right\| \\
& =l \sqrt{n} \max _{t \in T}\|e(t)\|+\frac{1}{\sqrt{n}}\|z\| .
\end{aligned}
$$

Taking into account (1), (6) can be written as follows:

$$
\begin{gathered}
\sum_{i=1}^{I} \max \left\{\sum_{t \subset T} x_{i}(t)-\sum_{t \in T} e_{i}(t), 0\right\} \\
\leqslant(l+1) \sqrt{n} \max _{t \in T}\|e(t)\|,
\end{gathered}
$$

which is condition (ii) of approximate value allocation for $\%$. Observe that since $x\left(t_{i}\right)$ is chosen arbitrarily to belong in the set $D\left(p, t_{i}\right)$ for all $i=1,2, \ldots, l$, then $x(t) \in D(p, t)$ for all $t \in T$. Since $x(t) \in D(p, t) \subset H(p, t)$ then $p \cdot x(t) \leqslant \operatorname{Sh}(p, t)$. Suppose that there exists $r>0$ such that $\operatorname{Sh}(p, t)-p \cdot x(t)=r$. By local nonsatiation there exists $x^{\prime}$ in $S(x(t), r) \cap \mathbb{R}^{\prime}$ such that $x^{\prime} \in H(p, t)$ and $x^{\prime}>_{t} x(t)$, but this contradicts maximality of $x(t)$ in $H(p, t)$. Hence, $p \cdot x(t)=\operatorname{Sh}(p, t)=M(x, p, t)$ which is condition (i) of the definition of approximate value allocation for $\mathscr{E}$. This completes the proof of the theorem.

Proof of Theorem 2. Consider an exchange economy with three agents and two commodities denoted by $T=\{1,2,3\}$ and $x, y$, respectively. Their utility functions and their initial endowments are given as follows:

$$
\begin{aligned}
& u_{1}\left(x_{1}, y_{1}\right)=\frac{1}{2} x_{1}+\frac{1}{2} y_{1}, \quad e_{1}=(0,0) . \\
& u_{2}\left(x_{2}, y_{2}\right)=\left(\frac{1}{2} \sqrt{x_{2}}+\frac{1}{2} \sqrt{y_{2}}\right)^{2}, \quad e_{2}=(0,4), \\
& u_{3}\left(x_{3}, y_{3}\right)=\left(\frac{1}{2} \sqrt{x_{3}}+\frac{1}{2} \sqrt{y_{3}}\right)^{2}, \quad e_{3}=(4.0) .
\end{aligned}
$$

A computation of the characteristic function $v_{u}$ gives:

$$
\begin{aligned}
v_{u}(\{1\}) & =0, \\
v_{u}(\{2\}) & =v_{u}(\{3\})=1, \\
v_{u}(\{1,2\}) & =2, \\
v_{u}(\{1,3\}) & =2, \\
v_{u}(\{2,3\}) & =v_{u}(\{1,2,3\})=4 .
\end{aligned}
$$

Moreover, we can compute the Shapley value of agent 1 and find that $\mathrm{Sh}_{1}=2 / 6$. Note that by group rationality we have that $v(\{1,2,3\})=$ $\sum_{i \in T} \mathrm{Sh}_{i}=4$. Since agents 2 and 3 have identical utility functions, they are 
treated symmetrically and thus we have that $\mathrm{Sh}_{2}=\mathrm{Sh}_{3}=11 / 6$. The value allocation is $\left(x_{1}, y_{1}\right)=(2 / 6,2 / 6), \quad\left(x_{2}, y_{2}\right)=\left(x_{3}, y_{3}\right)=(11 / 6,11 / 6)$. However, the above value allocation is not $c$-fair. In fact, note that for $S_{1}=\{1\}, S_{2}=\{2,3\}$ the assignment $\left(\bar{x}_{1}, \bar{y}_{1}\right)=(0,0),\left(\bar{x}_{2}, \bar{y}_{2}\right)=\left(\bar{x}_{3}, \bar{y}_{3}\right)=$ $(13 / 6,13 / 6)$ makes each member of coalition $S_{2}$ better off by obtaining the net trade of coalition $S_{1}$, i.e., $u_{i}\left(\bar{x}_{i}, \bar{y}_{i}\right)=u_{i}(13 / 6,13 / 6)=13 / 6>u_{i}\left(x_{i}, y_{i}\right)=$ $u_{i}(11 / 6,11 / 6)=11 / 6$ for $i \in S_{2}$ and $\left(\bar{x}_{2}, \bar{y}_{2}\right)-(0,4)+\left(\bar{x}_{3}, \bar{y}_{3}\right)-(4,0)=$ $\left(x_{1}, y_{1}\right)-(0,0)=(2 / 6,2 / 6)$.

Proof of Theorem 3. Apply Theorem 1.

\section{REFERENCES}

1. R. Anderson, M. Ali Khan, and S. Rashid, Approximate equilibria with bounds independent of preferences, Rev. Econ. Studies 44 (1982), 473-475.

2. J. Gabszewicz, Coalitional fairness of allocations in pure exchange economies, Econometrica 43 (1975), 661-668.

3. M. Ali Khan AND S. RASHID, Approximate equilibria in markets with indivisible commodities, J. Econ. Theory 28 (1982), 82-101.

4. Y. KanNaI AND R. Mantel, "Nonconvexifiable pareto sets, Econometrica 46 (1978), $571-576$.

5. L. W. McKenzIE, Demand theory without a utility index, Rev. Econ. Studies 24 (1956). 185-189.

6. L. ShaPLEY, Utility comparisons and the theory of games, in "La Decision." (Guilbaud. Ed.) Editions du CNRS, Paris, France, 1969.

7. L. Shapley AND M. Shubik, Quasi-cores in a monetary economy with non-convex preferences, Econometrica 34 (1966), 805-827.

8. W. Shafer, On the existence and interpretation of value allocation, Econometrica 48 (1980), 467-476.

9. R. STARR. Quasi equilibria in markets with non-convex preferences, Econometrica 17 (1969), 25-38.

10. N. C. YanNelis, Coalitional fairness in finite oligopolistic markets, mimeo. 